\title{
AFGL 2591 and Monoceros R2: Cavities in the Molecular Cloud
}

\author{
José M. Torrelles*, Paul T. P. Ho ${ }^{\dagger}$, Luis F. Rodríguez ${ }^{\ddagger}$, and Jorge Cantó ${ }^{\ddagger}$ \\ * Instituto de Astrofísica de Andalucia, CSIC \\ $\uparrow$ Harvard-Smithsonian Center for Astrophysics \\ \$nstituto de Astronomía, UNAM
}

We observed with the VLA(D) the $(\mathrm{J}, \mathrm{K})=(1,1)$ and $(2,2)$ inversion transition lines of ammonia toward two regions with molecular outflow: AFGL 2591 and Monoceros R2.

We detected a clumpy medium in AFGL 2591. Two of the detected clumps may be part of a shell-like structure surrounding the near IR bubble previously detected by Forrest and Shure (1986), and Lenzen (1987) (Figure 1, Torrelles et al. 1989a). By comparing the $(2,2)$ and $(1,1)$ lines we find that heating is more important for the eastern side of the shell-like structure, with temperatures of $\sim 200 \mathrm{~K}$ near to AFGL 2591 (Figure 2, Torrelles et al. 1989a). A significant enhanced emission in the $\mathrm{NH}_{3}$ line wings up to $\pm 3 \mathrm{~km} \mathrm{~s}^{-1}$ is observed near the peak temperature, indicating a local perturbation of the molecular gas. Our observations are consistent with the interpretation given by Forrest and Shure (1986) and Lenzen (1987) in terms that the near IR bubble is reflected light of a cavity, traced by the ammonia shell-like structure, and created in the molecular cloud by a central outflow probably related to AFGL 2591. The location of the radiocontinuum sources (1), (2), and (3) (Campbell 1984) on the edges of the neutral shell-like structure (see Figure 2) suggests that these could be bright rims externally excited by AFGL 2591 in a similar way as in Cepheus A (Torrelles et al. 1986).

The ammonia emission in Mon R2 shows a clumpy structure with multiple condensations. These condensations are mainly distributed in a remarkable arclike structure (Figure 3, Torrelles et al. 1989) located $\sim 40$ " SW of the "blyster" type HII region (Massi, Felli, and Simon 1985). Within this arc we find a velocity gradient with the highest velocities away from the stellar activity center. By comparing the $(2,2)$ and $(1,1)$ lines, we also find a temperature gradient, with the highest temperatures toward the stellar activity center (see Figure 3). These properties imply an interaction of the stellar activity center with the high-density molecular gas. We favour two possible explanations for the VLA ammonia arclike structure:

(1) It may be part of an inner molecular wall of a toroid's cavity created by the pressure of the stellar wind driving the molecular outflow. (A molecular toroid was suggested to be in Mon R2 to collimate the bipolar outflow in the SE-NW direction, Torrelles et al. 1983.) This model predicts the presence of another arclike structure located $\sim 40^{\prime \prime} \mathrm{NE}$ of the HII region. However we could not detect this counterpart structure in the present experiment due to primary beam response, 0.1 at this position.

(2) It may be produced by the expansion of the "blyster" type HII region in the champagne phase. In this case, a more complete molecular shell structure bordering the HII region may be present, but undetected in the present experiment by the primary beam response. 
We think that VLA ammonia observations with a new phase center are required to differentiate between models (1) and (2).

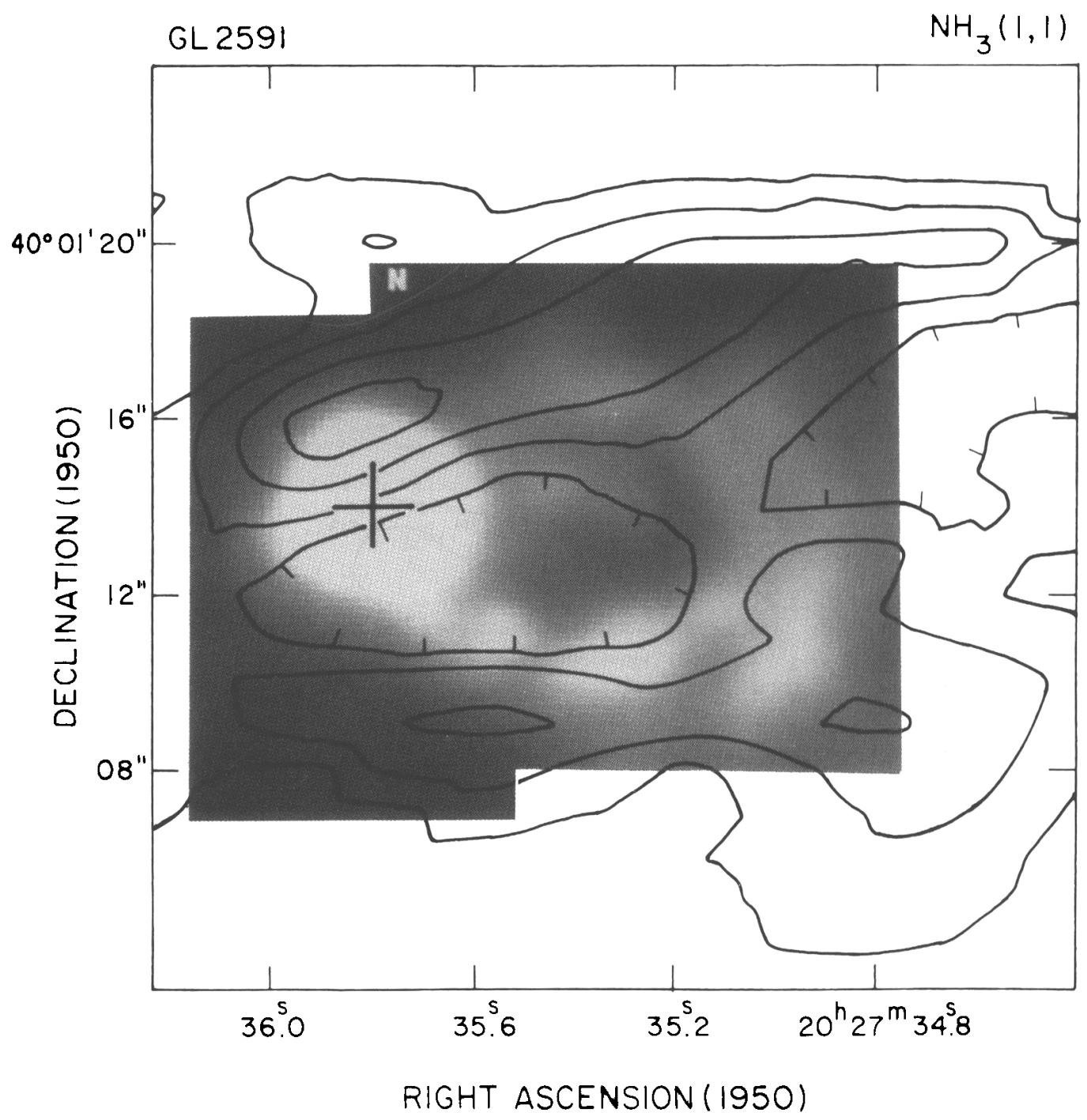

Figure 1. Contour map of the integrated $\mathrm{NH}_{3}(1,1)$ emission over the $-6.3 \rightarrow-4.5 \mathrm{~km} \mathrm{~s}^{-1}$ range (synthesized beam $=6^{\prime \prime}$ ) superposed on the $K$ image of Forrest and Shure (1986). Contour levels are $=-2,2,3,4,5,6 \times\left(30 \mathrm{mJy} /\right.$ beam $\left.\mathrm{km} \mathrm{s}^{-1}\right)$. Cross denotes the position of the AFGL 2591 source. (Figure adopted from Torrelles et al. 1989a.) 


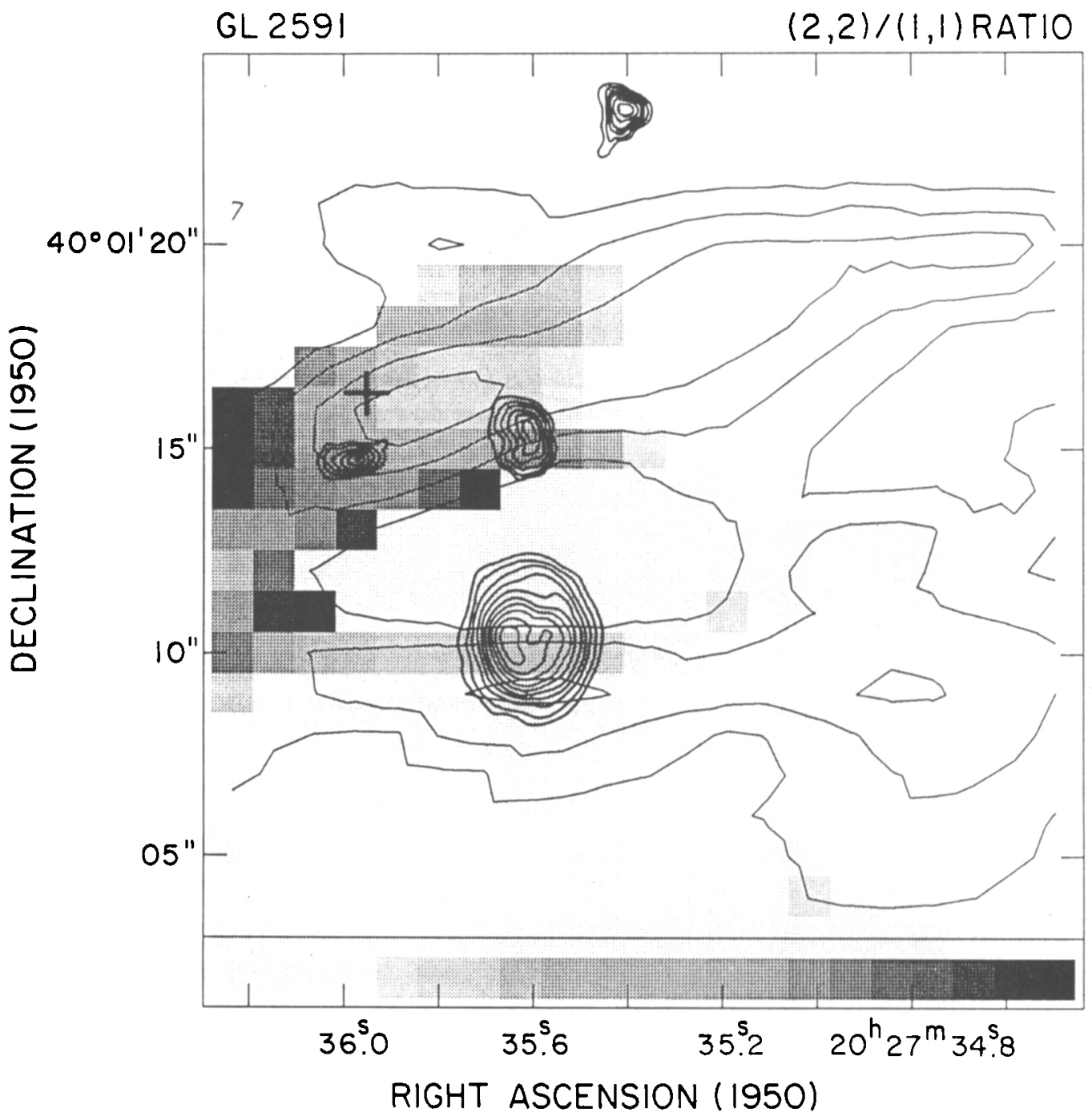

Figure 2. $\mathrm{S}_{\nu}(2,2 ; \mathrm{m}) / \mathrm{S}_{\nu}(1,1 ; \mathrm{m})$ ratio (grey scale) superposed to the $\mathrm{NH}_{3}(1,1)$ emission (Figure 1). The grey scale is linear and ranges from 0.6 to 3 for the flux ratio from left to right of the grey bar (down of figure). Radio continuum sources $(3,1,2,4$, in decreasing Right Ascension) from Campbell (1984) are also superposed. Cross denotes the $\mathrm{H}_{2} \mathrm{O}$ maser position (Wynn-Williams et al. 1977). (Figure adopted from Torrelles et al. 1989a.) 


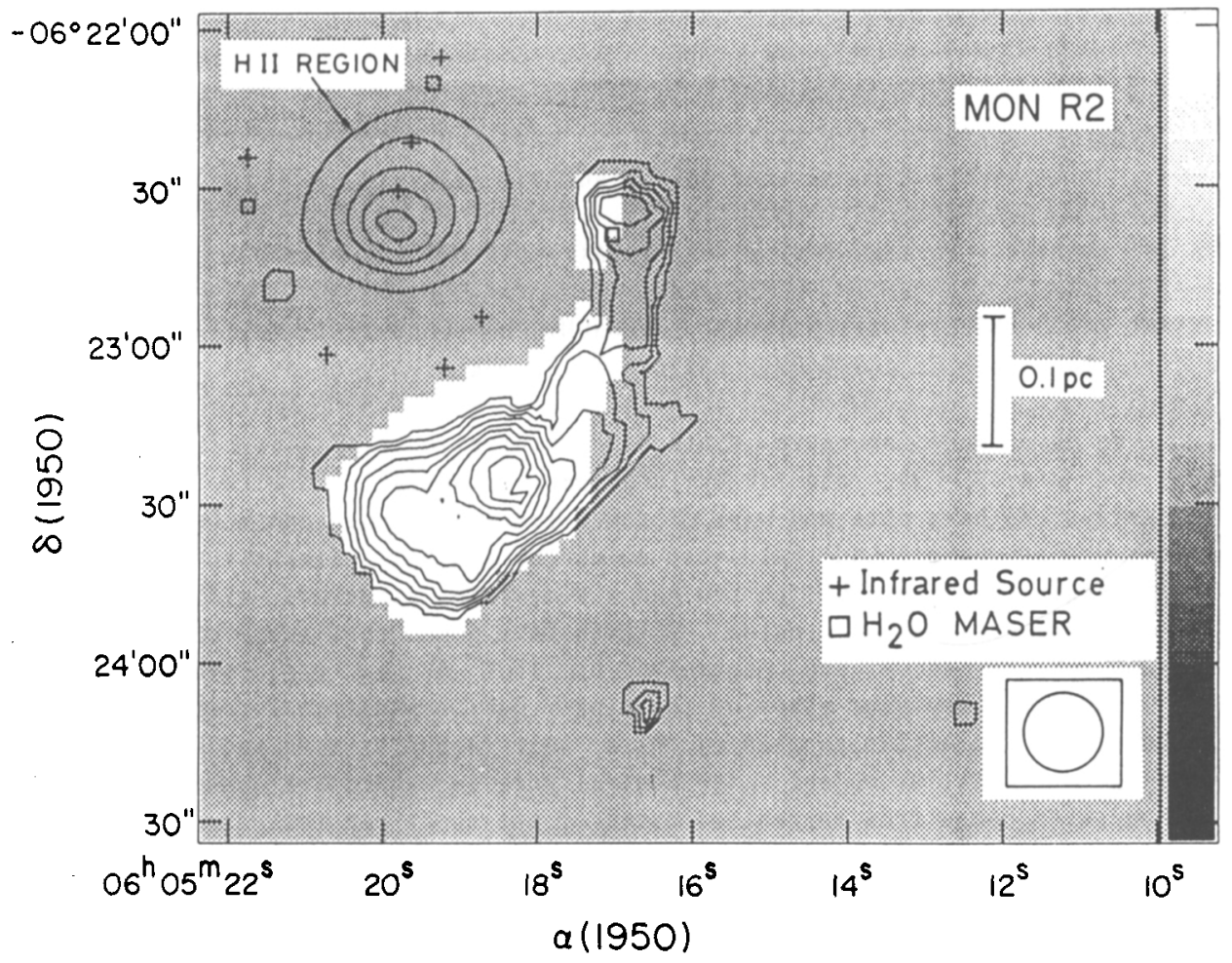

Figure 3. Contour map and grey scale image of the integrated intensity of the $(1,1)$ and $(2,2)$ ammonia main component line, respectively, over the $V_{L S R}=9.0 \rightarrow 12.6 \mathrm{~km} \mathrm{~s}^{-1}$ range (synthesized beam $=15^{\prime \prime}$ ). Contour levels are $=1,2,3,4,5,6,7,8,9$ $\times\left(43 \mathrm{mJy} /\right.$ beam $\left.\mathrm{km} \mathrm{s} \mathrm{s}^{-1}\right)$. The grey scale is linear and ranges from -43 to 43 $\mathrm{mJy} /$ beam $\mathrm{km} \mathrm{s}^{-1}$ from down to up of the grey bar (right of figure). Continuum map $(\lambda=1.3 \mathrm{~cm})$ of the "blyster" type HII region is also superposed. Note that the $(2,2)$ arclike structure is shifted to the $\mathrm{NE}$ of the $(1,1)$ one, indicating an important heating at that edge. (Figure adopted from Torrelles et al. 1989b.)

\section{References}

Campbell, B. 1984, Ap.J., 287, 334.

Forrest, W. J., and Shure, M. A. 1986, Ap. J. (Letters), 311, L81.

Lenzen, R. 1987, Astr. Ap., 173, 124 :

Massi, M., Felli, M., and Simon, M. 1985, Astr. Ap., 152, 387.

Torrelles, J. M., Ho, P. T. P., Rodríguez, L. F., and Cantó, J. 1989a, Ap. J., in press (July 15).

Torrelles, J. M., Ho, P. T. P., Rodríguez, L. F., and Cantó, J. 1989b, Ap. J., in press (November 15).

Torrelles, J. M., Ho, P. T. P., Rodríguez, L. F., and Cantó, J. 1986, Ap. J., 305, 721.

Torrelles, J. M., Rodríguez, L. F., Cantó, J. Carral, P., Marcaide, J., Moran, J., and Ho, P. T. P. 1983, Ap. J., $274,214$.

Wynn-Williams, C. G., Becklin, E. E., Forster, J. R,, Matthews, K., Neugebauer, G., Welch, W. J., and Wright, M. C. H. 1977, Ap. J. (Letters), 211, L89. 